\title{
Soil bacteria as a basis for sustainable development of the environment
}

\author{
Vasili Travkin ${ }^{1}$, Dilmurod Morudullaev ${ }^{1}$, Irina Artemyeva ${ }^{1}$, Nataliya Suzina $^{2}$, and Inna Solyanikova ${ }^{1,2,{ }^{*}}$ \\ ${ }^{1}$ Federal State Autonomous Educational Institution of Higher Education «Belgorod National Research University», 308015 Belgorod, \\ Russia \\ ${ }^{2}$ G.K. Skryabin Institute of Biochemistry and Physiology of Microorganisms, Pushchino Center for Biological Research of the Russian \\ Academy of Sciences, Pushchino, Moscow Region, 142290, Russian Federation
}

\begin{abstract}
Soil is an inexhaustible source of microorganisms of significant interest to biotechnology. Bacteria are able to control the growth of pathogenic microflora, stimulate plant growth, and decompose pollutants of varying degrees of toxicity. Bacteria make a significant contribution to the cycle of substances. The aim of this work was to isolate aerobic microorganisms from soil samples of two types - forest, without technogenic history, and agro-industrial, and to evaluate their properties. 15 strains of bacteria were isolated from forest soil, of which representatives of the genera Rhodococcus, Bacillus, Arthrobacter, Paenibacillus, Pseudomonas, Acinetobacter were able to degrade such persistent pollutants as chlorophenols, biphenyl and naphthalene. 77 strains were isolated from chernozem, of which 15 used benzoate as the sole source of carbon and energy. Thus, it has been shown that bacterial strains isolated from both forest and agricultural soil have a certain biodegradative activity, which allows them to reduce the level of pollution.
\end{abstract}

\section{Introduction}

The largest carbon storage on Earth is stored in forest soils [1]. Because forests are known to act as carbon sinks [2], they play an important role in the global carbon cycle and therefore influence climate. The main reservoir of carbon in forests is soil organic matter (SOM). Soil microorganisms play a crucial role in the transformation and decomposition of SOM [3].

Due to the fact that the main polymers of plants are lignin and cellulose, the forest can be considered as a natural factor contributing to the selection and fixation in the genome of microorganisms living in forest soil of specific genes associated with such functions as resistance to stressful conditions, including starvation, changes in $\mathrm{pH}$, temperature, the presence of toxic compounds, for example, chlorophenols, which are constituent fractions of lignin. Areas of research in soil microbiology: assessment of the general state of microbial systems, seasonal fluctuations in the biomass of the microbiocenosis, distribution of microorganisms of different taxonomic groups in soils of different types, study of the effect of anthropogenic load on changes in the number and diversity of soil microorganisms, study of rhizosphere microorganisms, including bacteria that stimulate plant growth (rhizosphere bacteria that promote plant growth). the features of the interaction of microorganisms with the host plant were revealed, mechanisms of the positive action of bacteria on plants, etc. In contrast, bacterial strains in agricultural soils are exposed to specific stressors such as pesticides, which suggests that they develop the ability to break down such compounds. On the other hand, interaction with plants allows an increase in the number of bacterial species belonging to the plant rhizosphere.

The aim of this work was to isolate aerobic microorganisms from soil samples of two types - forest, without technogenic history, and agro-industrial, and to evaluate their properties.

\section{Material and Methods}

\subsection{Microorganisms and cultivation conditions}

Microorganisms were isolated from soil taken in the vicinity of Pushchino, Moscow region (Russia), and from rhizosphere agricultural soil of the cities of Belgorod and Orel (Russia). In each case, three samples were taken from a depth of $5-10 \mathrm{~cm}$. The soil was used to isolate microorganisms after mixing the collected samples. The soil samples $(2 \mathrm{~g})$ were added to Erlenmeyer flasks with $100 \mathrm{~mL}$ of mineral medium of the following composition $(\mathrm{g} / \mathrm{L}): \mathrm{Na}_{2} \mathrm{HPO}_{4}-0.7$; $\mathrm{KH}_{2} \mathrm{PO}_{4}-0.5 ; \mathrm{NH}_{4} \mathrm{NO}_{3}-0.75 ; \mathrm{MgSO} 4 \cdot 7 \mathrm{H}_{2} \mathrm{O}-$ $0.2 ; \mathrm{MnSO}_{4}-0.001 ; \mathrm{FeSO}_{4}-0.02, \mathrm{NaHCO}_{3}-0.25$ containing $10 \%$ (vol/vol) Luria Bertani (LB) medium. The samples were cultivated on a shaker $(180 \mathrm{rpm})$ at $28^{\circ} \mathrm{C}$ for 2 days. Cells were seeded on LB agar-rich medium after dilution to $10^{-6}-10^{-8}$. Separate colonies differing in morphotype were transferred on a solid LB for further work.

\footnotetext{
Corresponding author: $\underline{\text { Solyanikova } @ \text {,bsu.edu.ru }}$
} 
The ability of strains to degrade various aromatic compounds as the only source of carbon and energy was assessed by sowing cells grown in a rich medium into a mineral medium with target substrates as a source of carbon. The concentration of growth compounds was as following (mg/l): phenol - 200; benzoate - 300; monochlorinated phenols - 50; di- and trichlorinated phenols - 20; monochlorobenzoates - 100; di- and trichlorobenzoates - 50; 2,4-dichlorophenoxyacetate 200, 2,4,5-trichlorophenoxyacetate - 150. Waterinsoluble substrates were added in the form of salts or solutions in dimethylformamide.

The ability of strains to transform trinitrotoluene (TNT) or hexogen (RDX) was studied by cultivating strains in Petri dishes with agar nitrogen-free mineral medium containing $50 \mathrm{mg} / \mathrm{l}$ TNT or RDX and Luria Broth (LB) 10\% (vol/vol). The grown cells were washed off with liquid nitrogen-free mineral medium and used as an inoculum $(10 \% \mathrm{vol})$ for inoculation of $750 \mathrm{ml}$ flasks with $100 \mathrm{ml}$ of medium, to which $50 \mathrm{mg} / \mathrm{l} \mathrm{TNT} / \mathrm{RDX}$ and LB $(5 \% \mathrm{vol} / \mathrm{vol})$ or succinate $(500 \mathrm{mg} / \mathrm{l})$. For biological control, cells were cultured under the same conditions without adding TNT / RDX to the medium. For chemical control, flasks with TNT / RDX and LB / succinate in mineral medium without inoculation were kept for the same time as in the experiment.

\subsection{Identification of bacteria and phylogenetic analysis}

The strains were identified based on an analysis of the 16S rRNA genes. The genomic DNA was isolated with the ZymoReseacher Quick-DNA Fungal/Bacterial Miniprep Kit (Zymo Reseach, United States) according to the manufacturer's recommendation. The $16 \mathrm{~S}$ rRNA gene was amplified by polymerase chain reaction (PCR) using universal primers for $16 \mathrm{~S}$ rRNA prokaryotes: $27 \mathrm{f}$ (5'-AGAGTTTGATCCTGGCTCAG-3') and 1525r (5'AAGGAGGTGATCCAGCC-3') [4]. PCR was performed on a My-Cycler, Tetrad 2 instrument (BioRad Laboratories, United States).

Primary phylogenetic screening of the obtained sequences was performed with the BLAST program [http://www.ncbi.nlm.nih.gov/blast] and the EzBioCloud database (www.ezbiocloud.net). For phylogenetic analysis, 16S rRNA gene sequences were taken from the GenBank database (www.ncbi.nlm.nih.gov). The obtained nucleotide sequences of the 16S rRNA gene were manually aligned with sequences of reference strains of the nearest microorganisms with the CLUSTAL W program [5].

\subsection{Bright microscopy}

Cell cultures were examined under a Nikon Eclipse Ci microscope (Nikon, Japan) equipped with a camera ProgRes Speed XT core5 (Jenoptik, Germany).

\subsection{Detection of antifungal activity}

The ability of the isolated strains to control the growth of phytopathogenic fungi was investigated by the method of perpendicular strokes. The method is based on perpendicular inoculation of a streak of a test culture of the fungus for a streak of isolated bacteria on solid Sabouraud medium [https://www.bd.com/resource.aspx?IDX=30529].

\section{Results and Discussion}

\subsection{Strain isolation and identification}

15 strains of bacteria were isolated from forest soil, of which representatives of the genera Rhodococcus, Bacillus, Arthrobacter, Paenibacillus, Pseudomonas, Acinetobacter. 77 strains of bacteria were isolated from field soil near Orel and Belgorod, represented by the genera Streptomycetes, Bacillus, Pseudomonas, Paenibacillus, Rhodococcus, Acinetobacter.

The method of light microscopy was used for the primary assessment of the species diversity of the isolated strains. Figure 1 shows phase contrast images of isolated microorganisms that differ in cell morphology (Fig. 1).

Among the newly isolated strains, higher actinomycetes with variously organized hyphae in the mycelium prevailed (Fig. 1, b, c, g, i, 1), many of which had the ability to synthesize colour pigments (Fig. 1, b, i). Strains of spore-forming bacteria with different cell morphology were also isolated (Fig. 1, d, f, h, j, k). Some of them also released coloured pigmen into the growth medium (Fig. 1, h). Rod-shaped non-spore-forming bacteria (Fig. 1, e) be found very rare, as were spherical or ovoid form bacteria (Fig. 1, a).

The variety of microorganisms inhabiting various ecosystems is determined by the conditions of their life and the characteristics of the environment [6]. For example, under conditions of salt stress, the bacterial community predominantly consisted of the types of Proteobacteria, Actinobacteria, Saccharibacteria, Chloroflexia, Acidobacteria, and Cyanobacteria [7]. Representatives of genera Pseudomonas, Stenotrophomonas, Rhodococcus, Acinetobacter are common representatives of soils contaminated with various xenobiotics $[8,9]$.

\subsection{The ability to decompose organic compounds}

All isolated strains were tested for their ability to use various aromatic compounds as the only growth substrate. Cultivation on an agar mineral medium containing xylene, toluene, and benzene as a growth substrate made it possible to select five strains resistant to these solvents. More than 10 cultures used benzoate as the only growth substrate.

Benzoate is one of the simplest aromatic compounds in structure. Great majority of microorganisms are capable of degrading benzoate in high concentrations, up 


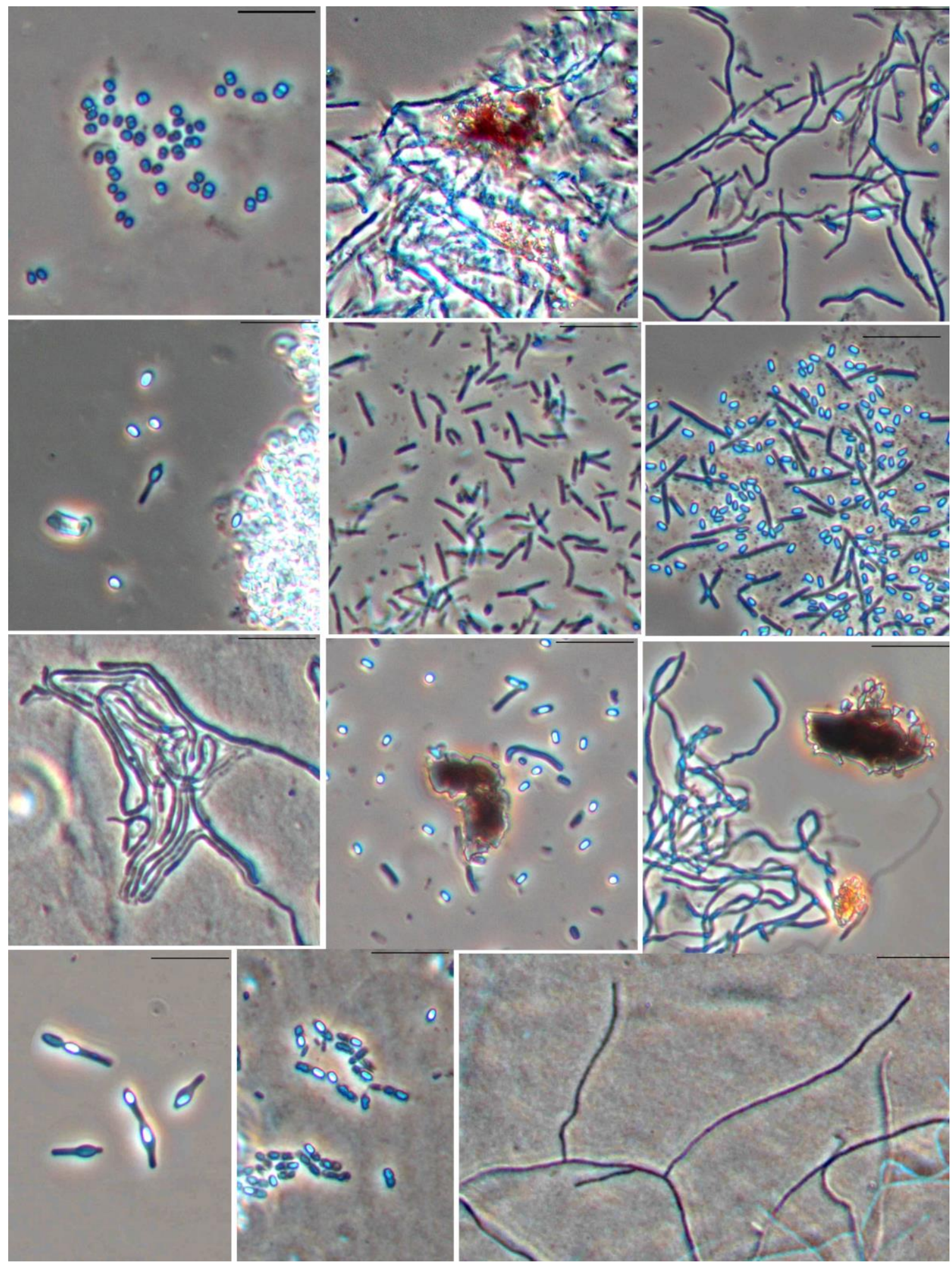

Fig. 1. The different morphotypes of prokaryotic cells forms isolated from the soil of Belgorod region (Bar - $10 \mu \mathrm{m})$.

to $2-5 \mathrm{~g} / 1$ [10]. Nevertheless, the presence of benzoate in the growth medium at a concentration above $0.75-1 \mathrm{~g} / \mathrm{l}$ has a negative effect on the maximum specific growth rate of cells.

Under aerobic conditions, the destruction of benzoate most often occurs with the formation of catechol [11]. The subsequent conversion of catechol depends on the type of dioxygenase that cleaves it. Catechol 1,2- dioxygenase breaks the aromatic ring between two carbon atoms bearing hydroxyl groups, resulting in the formation of cis,cis-muconic acid. In this case, the pathway of ortho-cleavage of catechol takes place.

If the aromatic ring of catechol is cleaved at position 2,3 under the action of catechol 2,3-dioxygenase, then the pathway of meta-cleavage of catechol operates [12]. 
In this case, hydroxymuconic semialdehyde is formed, which has a yellow color.

There is another way of aerobic degradation of benzoate, which involves the formation of hydroquinone. This pathway is extremely rare in microorganisms. Due to the oxidation of the resulting hydroquinone, the growth medium becomes dark in color. Thus, the presence of a specific color allows for an initial assessment of the pathways of benzoate degradation by microbial strains and the selection of the most interesting objects for further research. Cultivation of the isolated strains on agar mineral medium with benzoate in some cases led to the staining of the growth medium yellow or dark brown (Fig. 2).

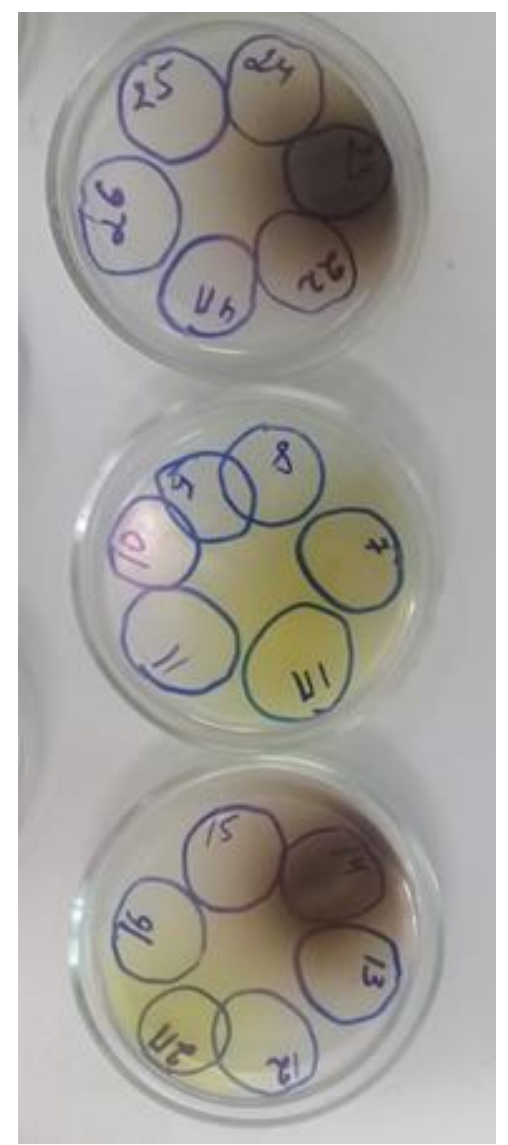

Fig. 2. Examples of staining of the growth medium as a result of the decomposition of benzoate with the formation of coloured intermediates.

\subsection{Transformation of explosives}

Generally, explosives like 2,4,6-trinitrotoluene (TNT), hexahydro-1,3,5-trinitro-1,3,5-triazine (RDX), and octahydro-1,3,5,7-tetranitro-1,3,5,7-tetrazocine (HMX) are used by armed forces as the energetic compounds of their choice [13]. As mentioned, there is a considerable undesirable environmental impact of both chemicals and toxic compounds of unexploded ordnance (UXO) and post-explosion by-products especially from industrial processes, military exercises and conflicts [13-14].

Strains of bacteria capable of transforming TNT and RDX were isolated from forest soil not contaminated with industrial waste (Fig. 3). Among the most active were bacteria of the genus Rhodoccus. This corresponds to the general ability of bacteria of this genus to decompose persistent pollutants [15].

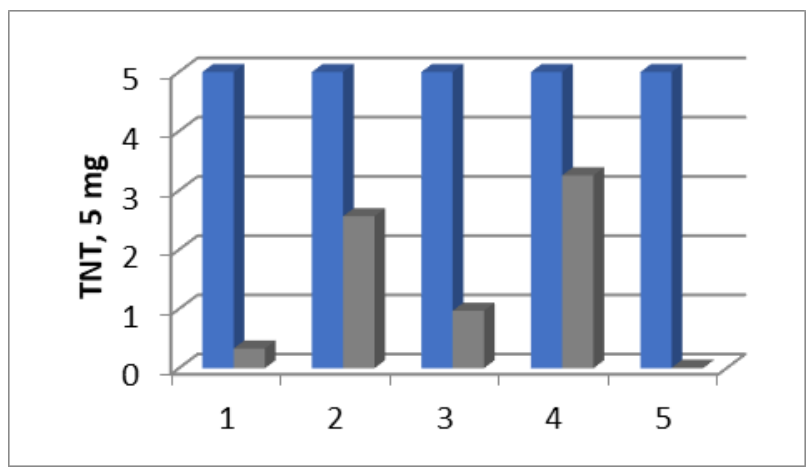

Fig. 3. Decrease of TNT (according to gas chromatography data) during incubation of strains VT5 (1), Rhodococcus sp. VT6 (2), Corynebacterium sp. VT2 (3), Rhodococcus sp. VT2 (4), $\gamma$-proteobacteria VT14 (5) in the medium with TNT (50 $\mathrm{mg} / \mathrm{l})$ and $\mathrm{LB}(5 \% \mathrm{vol} / \mathrm{vol})$ for 21 days.

RDX (hexahydro-1,3,5-trinitro-1,3,5-triazine) is a widely used explosive that has caused significant contamination at many military sites [16]. The Rhodococcus strain VT-7, isolated from clean forest soil, was capable of efficient transformation of RDX, as shown by the results of thin layer chromatography (TLC) (Fig.4).

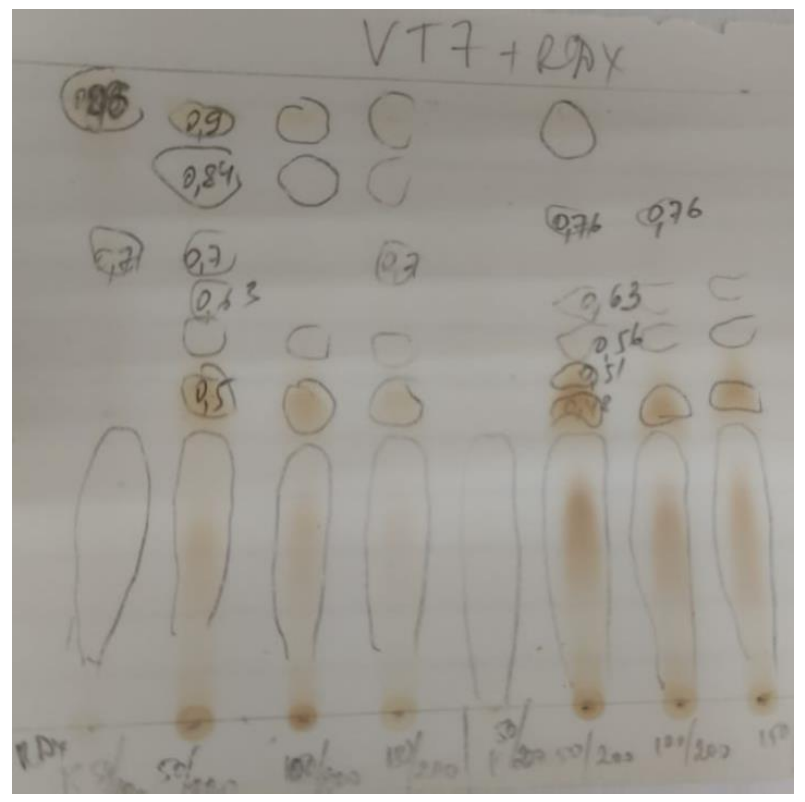

Fig. 4. Examples of biotransformation of RDX by strain Rhodococcus sp. VT-7, TLC.

RDX tends to be recalcitrant and can move from soil to surface waters or groundwater. Genes encoding enzymes for RDX degradation have been identified in isolates of the genera Rhodococcus, Gordonia, Williamsia (within the suborder Corynebacterineae, phylum Actinobacteria) and Microbacterium (suborder Micrococcineae, phylum Actinobacteria) [17]. 


\section{Conclusion}

Soil bacteria - destructors of persistent pollutants were isolated from both forest and agricultural soil. Some of them are effective antagonists of bacterial and fungal phytopathogens. Both biotechnologically significant strains and strains reflecting the microbial diversity of the soil microflora were identified. Further studies of their properties are ongoing. The obtained results show that ecosystems are capable of self-healing due to the presence of microorganisms with different degradative activity. This activity is not always determined by the set of compounds present in these econiches and may be the result of the transfer of genetic material between microorganisms or be the result of the action of enzymes in a wide substrate specificity.

This research was funded by RFBR according to the research project no. 19-54-80003.

\section{References}

1. T. Berger, C. Poyntner, R. Margesin, Folia Microbiologica, 66, 87-98 (2021)

2. P. Baldrian, Forest microbiome: diversity, complexity and dynamics. FEMS Microbiol. Rev., 41, 109-130 (2017) DOI: 10.1093/femsre/fuw040

3. L. Žifčáková, T. Vetrovsky, V. Lombard, B. Henrissat, A. Howe, P. Baldrian, Microbiome., 5, 122 (2017)

4. W.G. Weisburg, S.M. Barns, D.A. Pelletier, D.J. Lane, J. Bacteriol., 173 (2), 697-703 (1991)

5. J.D. Thompson, D.G. Higgins, T.J. Gibson, Nucleic Acids Res., 22 (22), 4673-4680 (1994)

6. Y.D. Jaffer, C.S. Purushothaman, H. Sanath Kumar, A.B. Irfan, P. Gireesh-Babu, P.A. Ganie, R.A.H. Bhat, A. Vennila, Arch. Microbiol., 201, 951-967 (2019) DOI: 10.1007/s00203-01901666-4

7. Y. Xu, G. Zhang, H. Ding, D. Ci1, L. Dai1, Z. Zhang, International Microbiology, 23, 453-465 (2020) DOI: 10.1007/s10123-020-00118-0

8. N.B. Sutton, A.A.M. Langenhoff, D.H. Lasso, B. van der Zaan, P. van Gaans, F. Maphosa, H. Smidt, T. Grotenhuis, H.H.M. Rijnaarts, Appl. Microbiol. Biotechnol., 98, 2751-2764 (2014) DOI: $10.1007 / \mathrm{s} 00253-013-5256-4$

9. L.B. Salama, O.S. Obayorib, M.O. Iloric, O.O. Amundc, Biocatal. Agricult. Biotechnol., 32, 101951 (2021)

10. I.P. Solyanikova, O.V. Borzova, E.V. Emelyanova, E.S. Shumkova, N.V. Prisyazhnaya, E.G. Plotnikova, Biokhimiya, Moscow, 81 (9), 986-999 (2016)

11. F. Abbasian, R. Lockington, M. Megharaj, R. Naidu, Appl Biochem Biotechnol., 178, 224-250 (2016) DOI: 10.1007/s12010-015-1881-y
12. D. Pérez-Pantoja, P. Leiva-Novoa, R.A.Donoso, C. Little, M. Godoy, D.H. Pieper, B. González, Appl. Environ. Microbiol., 81, 3914-3924 (2015) DOI: 10.1128/AEM.04207-14

13. S. Chatterjee, U. Deb, S. Datta, C. Walther, D.K. Gupta, Chemosphere, 184, 438-451 (2017)

14. P.T. Charles, A.A. Adams, J.R. Deschamps, et all, Sensors, 14, 4074-4085 (2014)

15. J. Zampolli, Z. Zeaiter, A. Di Canito, P. Di Gennaro, Appl. Microbiol. Biotechnol., 103, 1069-1080 (2019) DOI: 10.1007/s00253-0189539-7

16. E.L. Rylott, M.V. Budarina, A. Barker, A. Lorenz, S.E. Strand, N.C. Bruce, New Fhitologist, 192 (2), 405-413 (2011)

17. J.M. Collier, B. Chai, J.R. Cole, M.M. Michalsen, A.M. Cupples, Appl. Microbiol. Biotechnol., 103, 7161-7175 (2019) DOI: 10.1007/s00253-01910022-x 